\title{
DNA-functionalized dye-loaded polymeric nanoparticles: ultrabright FRET platform for amplified detection of nucleic acids
}

\author{
Nina Melnychuk and Andrey S. Klymchenko* \\ Laboratoire de Bioimagerie et Pathologies, UMR 7021 CNRS, Faculté de Pharmacie, Université de \\ Strasbourg, France
}

\begin{abstract}
Going beyond the limits of optical biosensing motivates exploration of signal amplification strategies that convert a single molecular recognition event into a response equivalent to hundreds of fluorescent dyes. In this respect, Förster Resonance Energy Transfer (FRET) with bright fluorescent nanoparticles (NPs) is an attractive direction, but it is limited by poor efficiency of NPs as FRET donors, because their size is typically much larger than the Förster radius $(\sim 5 \mathrm{~nm})$. Here, we established FRET-based nanoparticle probes that overcome this fundamental limitation by exploiting a phenomenon of giant light harvesting with thousands of strongly coupled dyes in a polymer matrix. These nanoprobes are based on 40-nm dye-loaded poly(methyl methacrylate-co-methacrylic acid) (PMMA-MA) NPs, so-called light-harvesting nanoantennas, which are functionalized at their surface with oligonucleotides. To achieve this functionalization, we developed an original methodology: PMMA-MA was modified with azide/carboxylate bi-functional group that enabled assembly of small polymeric NPs and their further $\mathrm{Cu}$-free click coupling with oligonucleotides. The obtained functionalized nanoantenna behaves as giant energy donor, where hybridization of target nucleic acid (encoding survivin cancer marker) with $\sim 23$ grafted oligonucleotides/Cy5-acceptors switches on/off FRET from $~ 3200$ rhodamine-donors of the nanoantenna, leading to 75-fold signal amplification. In solution and on surfaces at single-particle level, the nanoprobe provides sequence-specific two-color ratiometric response to nucleic acids with limit of detection reaching $0.25 \mathrm{pM}$. It displays unprecedented brightness for a FRET biosensor: it outperforms analogous FRET-based molecular probe by $>2000$-fold and QDot-605 by $~ 100$-fold. The developed concept of amplified sensing will increase orders of magnitude sensitivity of fluorescent probes for biomolecular targets.
\end{abstract}




\section{INTRODUCTION}

The problem of limited brightness of fluorescent dyes ${ }^{1}$ in biomolecular sensing stimulated development of amplification mechanisms that convert a single molecular recognition event into a response equivalent to hundreds of fluorescent dyes. ${ }^{2}$ Beyond strategies that multiply (chemically/enzymatically) the number of molecular species, ${ }^{3}$ concepts of direct amplification of dye emission have been developed. First one is based on conjugated polymers, where a large number of aromatic unites strongly coupled by pi-conjugation can efficiently transfer the excitation energy to a single energy acceptor. ${ }^{4}$ Second one uses plasmonic nanostructures (nanoantennas), where fluorescence of single molecules located in the "hot spots" can be amplified. ${ }^{5}$ Fluorescent nanoparticles (NPs), ${ }^{6}$ such as quantum dots (QDots), ${ }^{7}$ dye-doped silica, ${ }^{8}$ aggregation-induced emission, ${ }^{9}$ conjugated polymer $^{10}$ and dye-loaded polymer $^{11}$ NPs, being much brighter than organic dyes, are promising scaffolds for fabrication of biosensors with signal amplification. To convert fluorescent NPs into biosensors (nanoprobes), Förster Resonance Energy Transfer (FRET) is generally used, where the particle serves as energy donor, while energy acceptors with target-recognition units are grafted to its surface. ${ }^{12}$ However, to achieve direct signal amplification in this case, efficient FRET from the whole particle to a single acceptor should be achieved, which is a fundamental challenge because the particle size is generally much larger than the Förster radius $(\sim 5 \mathrm{~nm}) .{ }^{12 \mathrm{a}-\mathrm{c}, 13}$ For instance, in case of QDots, which are probably the most popular NPs for fabrication of biosensors, ${ }^{12 b}$ efficient FRET can be obtained only when significant amounts of FRET acceptors (10-50) are attached to their surface through a thinnest possible organic shell. ${ }^{12 a, 14}$ Dye-loaded polymeric NPs are particularly attractive alternative to QDots, ${ }^{11}$ because of their superior brightness, ${ }^{15}$ biocompatible/biodegradable matrix and low cytotoxicity. ${ }^{16}$ Very recently, following a concept used by nature in chlorophyll, ${ }^{17}$ we designed dye-loaded polymeric NPs operating as giant light-harvesting nanoantenna. ${ }^{18}$ Its $>10,000$ rhodamine dyes, assembled together by bulky counterions within in 60-nm poly(methyl methacrylate-co-methacrylic acid (PMMA-MA)-based nanoparticle, transferred efficiently the energy to few FRET acceptors inside the particle, leading to amplification of their emission $>1000$ fold. It surpassed plasmonics-based amplification values, ${ }^{19}$ and enabled first observation of single molecules in sunlight conditions. ${ }^{18}$ However, in order to transform them into probes for biomolecules, surface chemistry of these unique nanomaterials remains to be developed.

Ubiquitous nature of nucleic acids (NAs) makes them highly important targets for detection in biological research and medical diagnostics. ${ }^{6 c, 20}$ Their ultra-low concentrations in biological samples, below the detection limit of fluorescent molecular probes, require molecular multiplication techniques, notably polymerase chain reaction (PCR), a multi-step process that uses complex mixture of reagents, sophisticated equipment and well-trained staff. ${ }^{21}$ Chemists developed variety of fluorescent probes that can access much lower concentrations of NAs, up to few pM, but they also use molecular multiplication, based on enzymes ${ }^{3 b}$ or hybridization chain reactions. ${ }^{3 a, 22}$ To achieve direct one-step amplification, without molecular multiplication, cationic conjugated polymers were initially proposed, ${ }^{4 \mathrm{~b}}$ because they can report on sequence specific hybridization improving detection limits down to $\leq 10 \mathrm{pM}$ range. ${ }^{23}$ However, their limitations are non-specific interaction with other NA duplexes and proteins, and strong dependence on the ionic strength. ${ }^{24}$ Noble metal nanoparticles offered new opportunities in NA detection. ${ }^{6 c, 20 \mathrm{e}}$ Very recently, Tinnefeld and co-workers pioneered the amplified detection of NAs based on plasmonics nanoantenna positioned precisely in space with help of DNA origami. ${ }^{25}$ The $^{2}$ 
amplification achieved was 7.3 on average, although higher values were observed for some NPs. In this respect, our organic nanoantennas are expected to offer new possibilities.

We hypothesize that giant light-harvesting in the organic nanoantenna can be used to establish a concept of amplified FRET-based sensing, where a few nucleic acid hybridization events at the nanoantenna surface can trigger fluorescence response of thousands of dyes. To this end, a methodology of chemical functionalization of the PMMA-MA-based nanoantenna with oligonucleotides has to be developed first. Moreover, to achieve efficient FRET, the size of the nanoantenna particle should be optimal (40-50 nm) and the distance from its surface to the FRET acceptor should be minimized. However, small PMMAMA-based NPs bearing nucleic acids have not been reported to date. The problem is inefficiency of the direct coupling of hydrophobic polymers (including PMMA-MA derivatives) with oligonucleotides because of difficulty to solubilize together highly apolar and polar species, ${ }^{26}$ so that solid phase synthesis is required. ${ }^{26 a, 27}$ On the other hand, the examples of direct modification of polymeric NPs by oligonucleotides are limited to block-copolymers containing relatively large hydrophilic units with PEG linkers, ${ }^{28}$ which is incompatible with our FRET strategy. Based on our recent work, showing that a single charge per polymer is sufficient to assemble small NPs by nanoprecipitation, ${ }^{29}$ we expect that addition of a reactive group next to this charged group will enable preparation of functionalizable nanoantennas.

In the present work, we developed a robust approach to functionalize small $(<50 \mathrm{~nm})$ polymeric NPs with oligonucleotides and thus provided the first platform based on dye-loaded organic nanoantennas for amplified sensing of biomolecules. Due to light-harvesting principle, we achieved efficient FRET from $\sim 3200$ donor dyes inside nanoantenna to $\sim 23$ acceptors hybridized at the particle surface, leading to average signal amplification of 75. Being $\sim 100$-fold brighter than QDot-605, the nanoprobes produce donor/acceptor emission ratio response to just 3-4 NA hybridizations per particle, which makes them the brightest nanoprobes reported to date. The nanoprobe was designed to detect a fragment of nucleic acid encoding survivin protein, an important anti-apoptotic cancer marker. ${ }^{30}$ The obtained nanoprobe operates in solution and on surfaces delivering limit of detection for the NA target of 5 and $0.25 \mathrm{pM}$, respectively, which is 1000-10,000 fold lower than that achieved using molecular probes. Given the universality of FRET-based detection concept, our functionalizable organic nanoantennas open the route to a broad range of ultrabright probes for amplified detection of biomolecules.
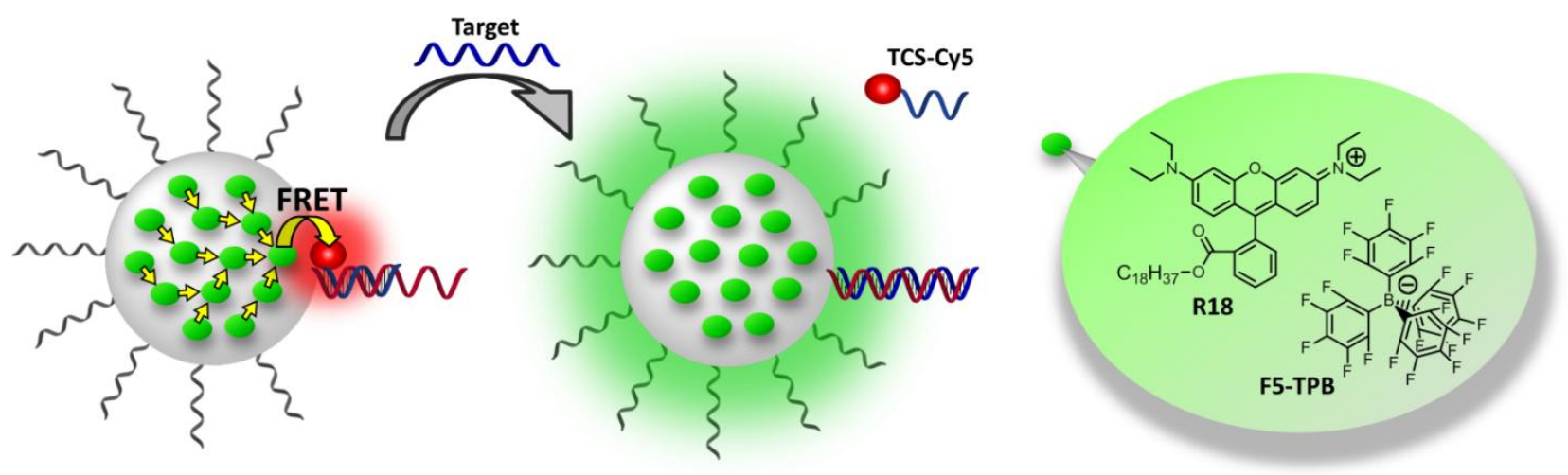

Figure 1. Concept of polymeric nanoantenna for amplified detection of nucleic acids. The dye-loaded nanoparticle is schematically presented, bearing non-coding (in gray) and capture (dark red) oligonucleotides. Yellow arrows schematically show the light-harvesting process toward FRET acceptor 
at the surface of nanoparticle. Target NA displaces short oligonucleotide with FRET acceptor (TCSCy5) from the NP surface, resulting in the FRET loss, so that the emission of the probe switches from red (acceptor) to green (donor). Chemical structures of the rhodamine derivative R18 and its bulky counterion F5-TPB are also shown.

\section{RESULTS AND DISCUSSION}

Design of nanoprobes for nucleic acids. In our design, the light-harvesting nanoantenna (FRET donor) is based on poly(methyl methacrylate-co-methacrylic acid (PMMA-MA, 1.6\% methacrylic acid) NPs loaded with ion pair of hydrophobic rhodamine (R18) with bulky hydrophobic counterion tetrakis(pentafluorophenyl)borate (F5-TPB). The latter serves as spacer between dyes to minimize their aggregation-caused quenching inside $\mathrm{NPs}^{16 \mathrm{a}}$ and ensures ultrafast excitation energy migration required for efficient light harvesting (Figure 1). ${ }^{18}$ This nanoantenna is modified at the surface with a capture oligonucleotide, complementary to a nucleic acid fragment encoding cancer marker survivin (20mer, SurC). SurC is hybridized with a short target-competitive sequence bearing FRET acceptor Cy5 (12mer, TCS-Cy5), so that the acceptor is localized close to the nanoantenna surface. Cy5 dye was selected because its absorption spectrum has a good spectral overlap with the emission spectrum of R18/F5TPB-loaded NPs with a Förster radius of $6.1 \mathrm{~nm} .{ }^{18}$ Combination of these two oligonucleotides was originally proposed by Mirkin and co-workers in NanoFlares, ${ }^{20 e, 31}$ which ensured quenching of dyes located close to gold NPs. In our case, this design is used to induce FRET form nanoantenna to Cy5 acceptor. Here, thousands of rhodamine dyes inside nanoantenna pump the energy to a few Cy5 acceptors at the surface, resulting in strong acceptor (red) emission amplified by the light harvesting (Figure 1). Then, the target nucleic acid displaces TCS-Cy5 acceptors and blocks FRET, so that nanoprobe should switch to green emission of nanoantenna. Importantly, a few hybridization events are expected to switch emission of thousands of dyes, provide this basis for signal amplification. To obtain such a nanoprobe, the primary challenge is to functionalize our polymeric nanoantenna with controlled number of oligonucleotides.

DNA-modified polymeric nanoparticles. In the first approach, we modified PMMA-MA polymer with an azide group (PMMA-N3), which was further reacted with oligonucleotide bearing dibenzocyclooctyne (DBCO) unit through a $\mathrm{Cu}$-free click cycloaddition. ${ }^{32}$ The latter was reported to provide efficient coupling without DNA damage. ${ }^{33}$ However, this direct coupling failed because we could not find an appropriate solvent to solubilize both polar oligonucleotide and apolar polymer. Moreover, in contrast to PMMA-MA, nanoprecipitation of PMMA-N3 gave only very large aggregates (Figure S1), because charged carboxylate group, essential for obtaining small NPs, ${ }^{18,29}$ was absent in PMMA-N3. Therefore, we designed a polymer bearing both charged carboxylate and reactive azide groups in close proximity. In addition to formation of small NPs, this additional charged group can favor the exposure of reactive azide group at the NPs surface (Figure 2). For this purpose, we developed a three-functional linker containing: (i) carboxyl as a charged group; (ii) azide for coupling with the oligonucleotide and (iii) amino group for conjugation with the polymer (Figure 2). As a building block, we chose the bi-protected aspartic acid, commonly used for solid-phase peptide synthesis, Fmoc$\mathrm{Asp}(\mathrm{OtBu})-\mathrm{OH}$. Its carboxylic function was modified with 3-chloropropanamine, and then the chlorine was substituted with an azide, which resulted also in Fmoc removal giving target linker Asp(OtBu)-N3 
(Figure 2). The latter was coupled with PMMA-MA through an amide bond. NMR spectra confirmed good yield of the polymer modification. Finally, tert-butyl group was removed giving a final polymer (PMMA-AspN3) for preparation of functionalized nanoantennas (Figure 2).

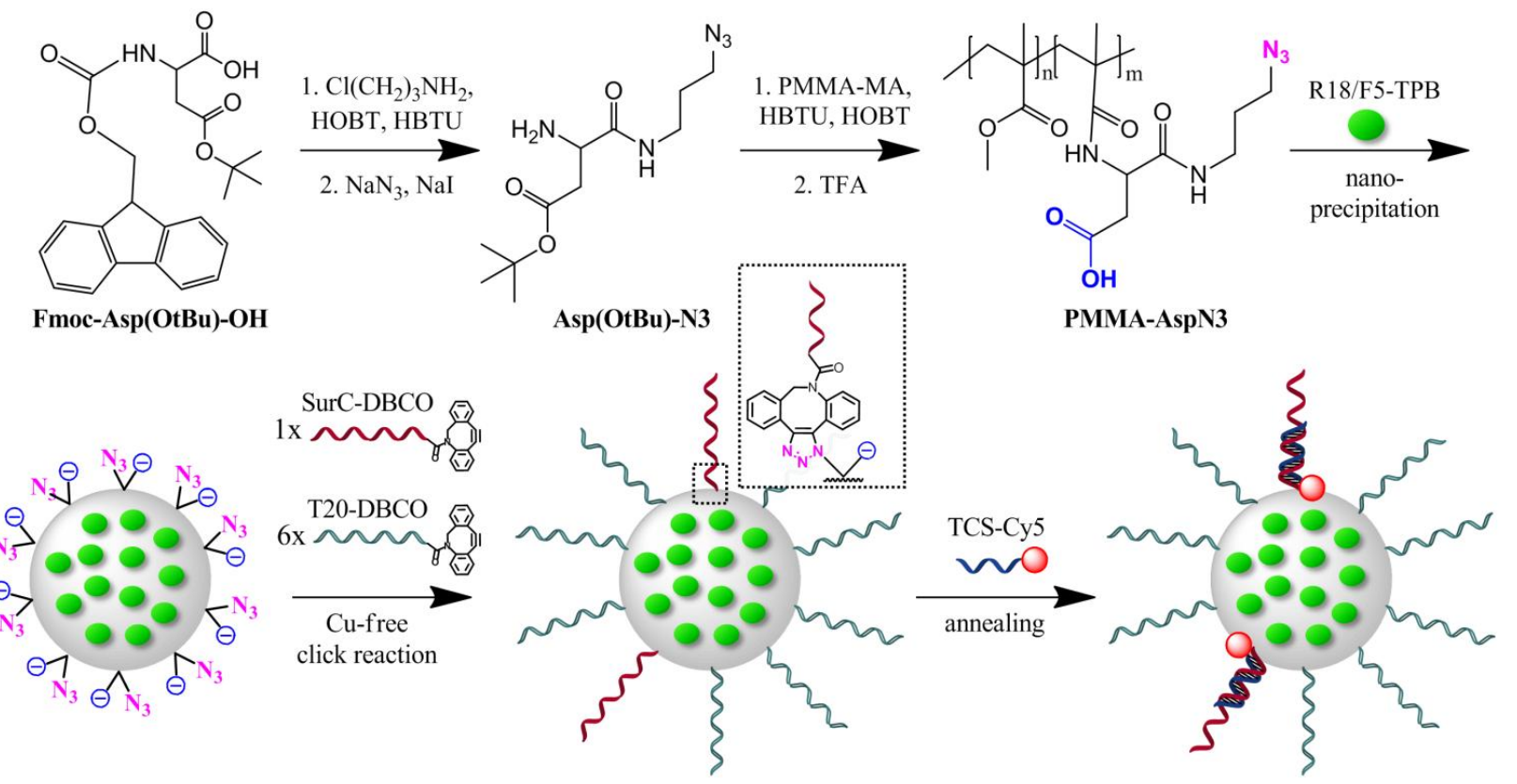

Figure 2. Scheme of synthesis of nanoprobes for nucleic acids. It includes synthesis of linker molecule $(\mathrm{Asp}(\mathrm{OtBu})-\mathrm{N} 3)$, its conjugation to PMMA-MA and deportation affording target polymer PMMAAspN3, nanoprecipitation of PMMA-AspN3 to obtain NPs bearing azide groups, DNA grafting to the surface of NPs and hybridization with Cy5-TCS.

Nanoprecipitation of acetonitrile solution of PMMA-AspN3 polymer with fluorescent dye (R18/F5TPB) into aqueous buffer ( $\mathrm{pH} 7.4)$ gave small NPs (35 nm, Figure 3a), highlighting the crucial role of the additional charged carboxylate group. Moreover, with modified nanoprecipitation protocol, concentration of PMMA-AspN3 NPs was further improved 10-fold (Figure S2), while keeping small particle size (42 nm, Figure 3a). Despite high 30 wt\% loading of R18/F5-TPB (e.g. 3200 dyes per 40 $\mathrm{nm}$ particle), the fluorescence quantum yield of NPs was very high (47\%, Table S1), which renders our nanoantenna particles exceptionally bright.

For grafting oligonucleotides, the obtained concentrated dye-loaded NPs were reacted with DBCObearing oligonucleotide SurC-DBCO for $18 \mathrm{~h}$ at different temperatures, followed by annealing with shorter oligonucleotide TCS-Cy5 bearing FRET acceptor. The purification of the DNA-conjugated NPs from non-reacted oligonucleotides was achieved by successive filtrations through $100 \mathrm{kDa}$ filters, as verified by absorption spectroscopy (Figure S3). Fluorescence spectra suggested that at 4 and $20^{\circ} \mathrm{C}$ the reaction did not occur, while at $40^{\circ} \mathrm{C}$ we observed a clear FRET signal from NPs to hybridized TCS$\mathrm{Cy} 5$, indicating successful conjugation reaction (Figure S4). Moreover, grafting reaction at $40^{\circ} \mathrm{C}$ was confirmed by absorption spectroscopy, where the signal of the hybridized TCS-Cy5 was observed in the purified samples, in contrast to negative control based on PMMA-MA NPs without azide group mixed with SurC-DBCO (Figure 3b). The heating at $40^{\circ} \mathrm{C}$ is probably necessary in order to increase the probability of collision between DBCO group grafted to oligonucleotide and azide group at the particle surface, in line with relatively slow DBCO/azide reaction kinetics reported recently for other type of 
polymeric NPs. ${ }^{28 \mathrm{c}}$ However, in phosphate buffer containing $12 \mathrm{mM} \mathrm{Mg}^{2+}$ ions, required for formation of stable duplexes, ${ }^{25}$ DNA-NPs conjugates (NP-SurC) showed relatively large size (Figure 3a), probably because of partial aggregation of the obtained NPs.

Stability of DNA-NPs conjugates can be improved by increasing number of oligonucleotides per particle, as it was recently shown for NPs built of ring-opening metathesis block copolymers. ${ }^{28 a}$ However, to achieve the highest optical amplification through the light-harvesting mechanism, minimal ratio of acceptors to donors should be used, ${ }^{18}$ i.e. minimal number of FRET acceptors should be grafted to the particle surface. To address these both issues, our NPs were reacted with a mixture of coding (SurC-DBCO) and non-coding (T20-DBCO) oligonucleotides. According to absorption spectroscopy, addition of T20-DBCO $(20 \mu \mathrm{M})$ to SurC-DBCO $(3 \mu \mathrm{M})$ did not inhibit the grafting of SurC to NPs surface (Figure $3 \mathrm{~b}$ ), probably because azide groups of NPs were in excess $(26 \mu \mathrm{M})$. Fluorescence spectra of these NPs (NP-SurC-T20/Cy5) showed strong emission of the FRET acceptor TCS-Cy5 around $670 \mathrm{~nm}$ (Figure 3c). Moreover, the emission maximum of Cy5 shifted to the red and its anisotropy value increased compared to free TCS-Cy5 (Figure S5), confirming the TCS-Cy5/SurC hybridization. These results suggested successful grafting of SurC-DBCO to NPs in the presence of T20-DBCO. Remarkably, NP-SurC-T20/Cy5 particles remained small in phosphate buffer with $\mathrm{Mg}^{2+}$ ions and their size did not change even after 2 month incubation in this medium (Figure 3a). Moreover, their emission spectrum with characteristic FRET signal remained practically invariant for this 2 month period (Figure S6). Therefore, grafting excess of non-coding DNA (T20) is essential for stability of our polymeric NPs. In addition, transmission electron microscopy (TEM) confirmed that conjugation of NPs with oligonucleotides did not modify their spherical shape and monodispersity, while their size increased only by $c a 5 \mathrm{~nm}$ (Figure 3d-g).
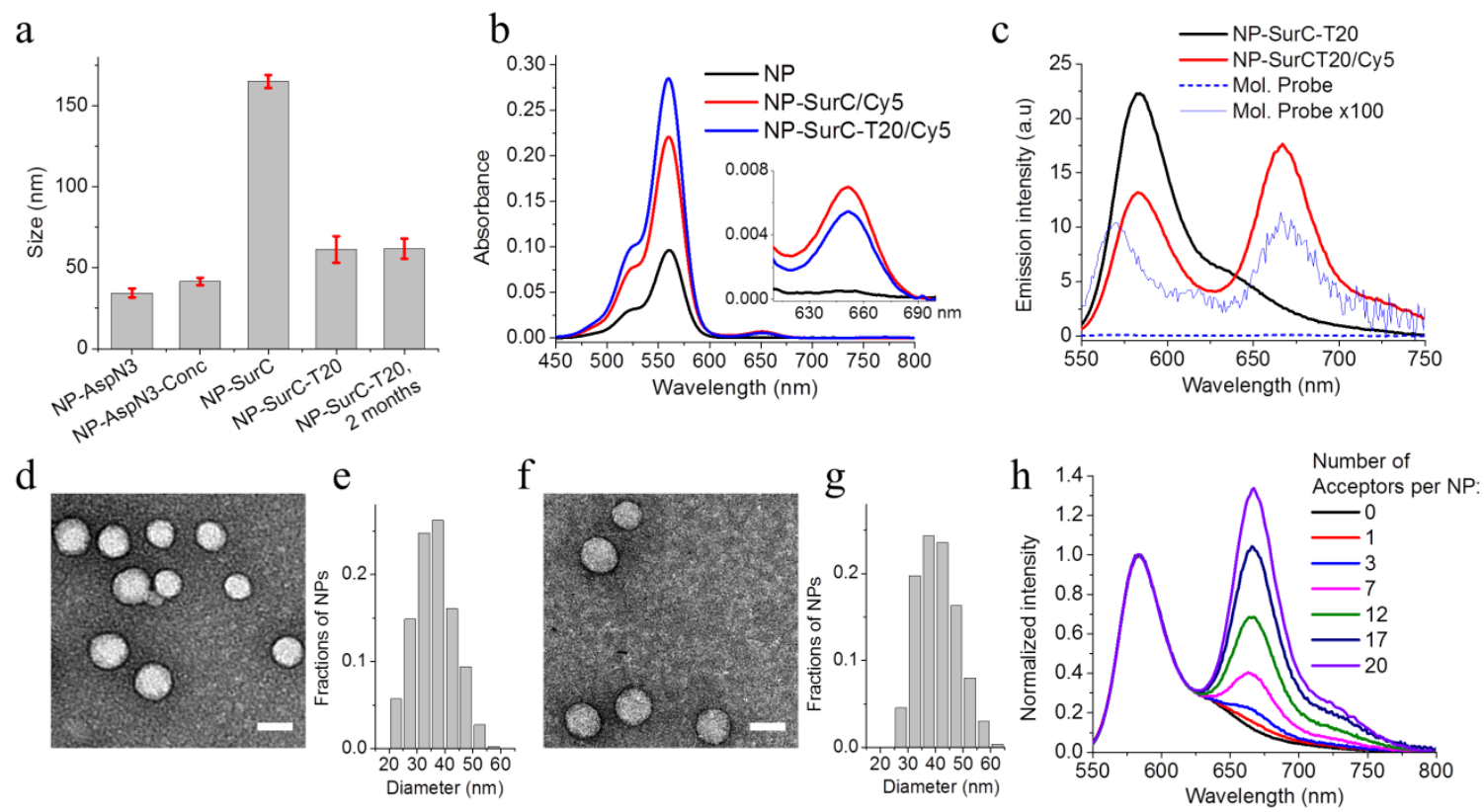

Figure 3. Characterization of DNA conjugates with nanoantenna particles. (a) Size by DLS of NPs bearing the motif AspN3 (NP-AspN3), NPs bearing AspN3 prepared at high concentration (NP-Asp-N3Conc), NPs bearing AspN3 and target-specific oligonucleotide SurC (NP-SurC), NPs bearing Asp-N3, SurC and non-specific oligonucleotide T20 (NP-SurC-T20), and the latter after 2 months storage (NPSurC-T20, 2 months). (b) Absorption spectra of control PMMA-MA NPs (without azide groups) and 
NP-AspN3 after reaction with SurC followed by hybridization with TCS-Cy5 (NP and NP-SurC/Cy5, respectively), and NP-AspN3 after reaction with SurC and T20 followed by hybridization with TCSCy5 (NP-SurC-T20/Cy5). (c) Fluorescence spectra of NP-SurC-T20 (43 pM) without and with TCSCy5 (1 nM) and comparison with spectra of corresponding molecular probe Cy3-SurC/TCS-Cy5 (1 nM) at the same instrumental conditions. For better comparison the latter spectrum was also multiplied 100fold, showing that it is $>100$ fold less bright than the NP-probe. TEM images of NP-AspN3 (d) and NPSurC-T20 (f) and corresponding size distribution statistics (e,g). Scale bar $50 \mathrm{~nm}$. (h) Normalized fluorescence spectra of NP-SurC-T20 at different number of hybridized TCS-Cy5 per particle. $20 \mathrm{mM}$ phosphate buffer ( $\mathrm{pH} 7.4$ ) with $30 \mathrm{mM}$ of $\mathrm{NaCl}$ and $12 \mathrm{mM}$ of $\mathrm{MgCl}_{2}$ was systematically used. (c,h) Excitation wavelength was $530 \mathrm{~nm}$.

Signal amplification by nanoantenna. Our further studies were focused on the most promising NPs bearing both SurC and T20. Their hybridized form with FRET acceptor TCS-Cy5 (NP-SurC-T20/Cy5) was called "NP-probe". The synthesis of these nanoprobes was repeated four times showing good reproducibility of their size and spectroscopic properties (Table S2). Based on the absorption data and the particle size from TEM $(41 \pm 12 \mathrm{~nm})$ we estimated that $23 \pm 3$ (s.e.m. n=4) FRET acceptors (TCSCy5) were grafted per nanoantenna particle containing $3200 \pm 400$ donor dyes. In this respect, two outstanding features of NP-probe should be mentioned: (i) high fluorescence quantum yield (46\%, Table S1) and (ii) $60 \pm 6 \%$ FRET efficiency from 3200donor dyes inside nanoantenna of 20-nm radius to 23 acceptors at the surface, i.e. through distances far beyond the Förster radius $(6.1 \mathrm{~nm})$. This is a highly efficient light-harvesting phenomenon with minimal energy losses, where giant ensemble of donors "pumps" the energy to few acceptors, which implies strong amplification of the acceptor emission (antenna effect). The antenna effect, measured as an intensity ratio of the donor to acceptor in the excitation spectra (Figure S7), ${ }^{34}$ was $58 \pm 1$ with high reproducibility for all four preparations (Table S2). This result means that excitation via nanoantenna amplifies acceptor (TCS-Cy5) emission 58-fold. To show the power of our nanoantenna amplification approach, we compared the NP-system with a molecular FRET probe (Cy3-SurC/TCS-Cy5), where SurC conjugate with Cy3 dye was hybridized with TCS-Cy5. The Cy3 dye was chosen because it exhibited similar absorption and emission properties to rhodamine dye used in our NPs (Table S1). Remarkably, for the same FRET acceptor concentration (1 $\mathrm{nM}$ of TCS-Cy5), the signal from our NP-probe was $>100$-fold higher compared to the molecular probe (Figure 3c), in line with the antenna effect values. As the NP-probe concentration in this case was 43 pM, the single NP-probe was $>2,000$-fold brighter than the single molecular probe. Finally, we verified the minimal number of TCS-Cy5 that NP-probe can distinguish by its FRET signal. To this end, TCSCy5 was hybridized with SurC-modified NPs at the different ratios (Figure S8). The increase in number of FRET acceptors per NP improved the FRET signal (Figure 3h). Remarkably, hybridization of 3-5 TCS-Cy5 per particle produced clearly detectable change in the dual emission of the nanoprobe, indicating that just a few molecular recognition events can control emission of $\sim 3200$ donor dyes inside nanoantenna. This unique sensitivity of our nanoantenna-based probes to few hybridization events together with strong signal amplification phenomenon are of key importance for detection of the target nucleic acids.

Detection of target nucleic acids. In the presence of the NA target encoding survivin NP-probe totally lost FRET signal at $670 \mathrm{~nm}$, in contrast to the control sample without the target (Figure 4a). Importantly, 
the response is ratiometric, displaying $>5$-fold change in the $\mathrm{A} / \mathrm{D}$ intensity ratio. This result shows that the target sequence displaced FRET acceptor TCS-Cy5 at NPs surface, thus stopping FRET. Moreover, the response of NP-probe decreased drastically already for a single mismatch in the target sequence, which was located in the middle of the region corresponding to the target-competitive sequence. Second and third mismatches further deteriorated the response to the oligonucleotide (Figure 4a, Figure S9), showing very good sequence-specificity of the NP-probe. We further verified the importance of the $\mathrm{Mg}^{2+}$ ions in the response of NP-probe. Remarkably, below $12 \mathrm{mM}$ of $\mathrm{Mg}^{2+}$, NP-probe showed a decrease in the FRET signal after 3h incubation without the target (Figure S10a), confirming that 12 $\mathrm{mM}$ concentration of $\mathrm{Mg}^{2+}$ ensures stability of the duplex between the capture sequence and TCS-Cy5. Importantly, the response of NP-probe to the target also decreased at lower $\mathrm{Mg}^{2+}$ concentrations (Figure $\mathrm{S} 10$ ), indicating that $\mathrm{Mg}^{2+}$ could also assist the displacement of TCS-Cy5 by the target sequence. Therefore, $12 \mathrm{mM}$ concentration of $\mathrm{Mg}^{2+}$ was systematically used in all further experiments. Then, the NP-probe diluted to TCS-Cy5 concentration of 10 pM (i.e. $~ 0.4$ pM NP-probe) was tested in growing concentrations of NA target. The relative intensity of the FRET acceptor, expressed as semi-empirical parameter of FRET efficiency, A/(A+D), ${ }^{35}$ gradually decreased in the concentration range of the NA target from 20 to $200 \mathrm{pM}$ (Figure 4b,c). The estimated limit of detection (LOD) was $5 \mathrm{pM}$, which is remarkably low for a standard fluorometer. Then, we checked whether our nanoprobe is operational in different biological media. In all studied media, namely phosphate buffered saline (PBS), PBS with bovine serum albumin (BSA), Opti-MEM (cell culture medium without serum), Opti-MEM with fetal bovine serum (FBS), and Opti-MEM with human serum, the probe showed robust ratiometric response to the NA target: decrease in the relative intensity of the acceptor emission (Figure 4d). Moreover, the probe preserved the response in the excess of the denatured calf thymus DNA, which served as model of random NA sequence. Thus, our NP-probe is compatible with highly complex media containing variety of biomolecules, including proteins and NAs.
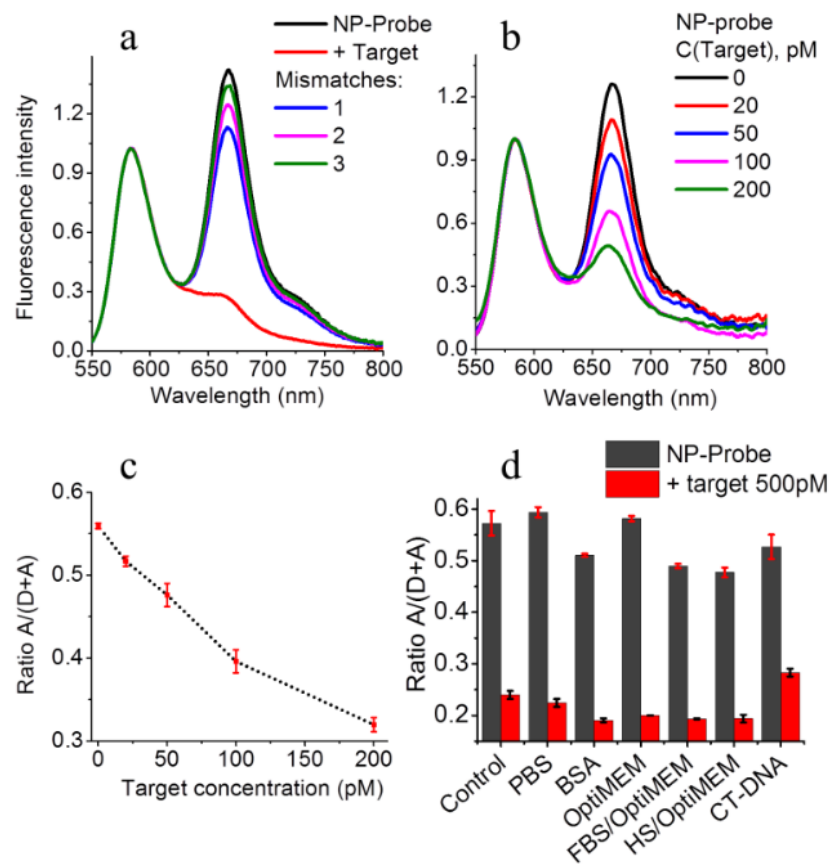

Figure 4. Response of nanoprobe to the nucleic acid target. (a) Fluorescence spectra of NP-probe (100 $\mathrm{pM}$ of TCS-Cy5) after incubation for $20 \mathrm{~h}$ at $4{ }^{\circ} \mathrm{C}$ without and with survivin NA target (1 nM). Targets 
containing 1, 2 and 3 mismatches were tested at the same conditions. Fluorescence spectra (b) and values of FRET response $\mathrm{A} /(\mathrm{A}+\mathrm{D})$ (c) of NP-Probe after incubation with target at different concentrations. A and D are the pick intensities of the acceptor (at $665 \mathrm{~nm}$ ) and the donor (at $580 \mathrm{~nm}$ ), respectively. Concentration of the NP-probe corresponds to $10 \mathrm{pM}$ of TCS-Cy5. $20 \mathrm{mM}$ phosphate buffer ( $\mathrm{pH}$ 7.4) with $30 \mathrm{mM}$ of $\mathrm{NaCl}$ and $12 \mathrm{mM}$ of $\mathrm{MgCl}_{2}$ was systematically used. (d) FRET response of the NP-probe (100 pM of TCS-Cy5) to the NA target $(500 \mathrm{pM})$ in different biological media (concentration of $\mathrm{Mg}^{2+}$ was adjusted to $12 \mathrm{mM}$ ): Control $(20 \mathrm{mM}$ phosphate buffer with $30 \mathrm{mM}$ of $\mathrm{NaCl}), \mathrm{PBS}, 10 \mu \mathrm{g} / \mathrm{mL}$ in PBS), BSA $(0.5 \mathrm{mg} / \mathrm{mL}$ in PBS), OptiMEM, FBS (inactivated, $10 \%$ in OptiMEM), Human Serum (inactivated, 10\% in OptiMEM) and DNA from calf thymus (CT-DNA, heat denatured. $(c, d)$ Error bars are standard deviation of the mean $(n=3)$. Excitation wavelength was 530 nm.

Evaluation of NP-probe at the single-particle level. The ultimate test for the performance of the nanoprobe is to verify whether it can operate at the level of single particle. To this end we modified the glass surface with A20 sequence, which is complementary to that of non-coding T20 sequence of our NP-probe (Figure 5a). After incubation with the NP-probe fluorescent particles were clearly seen displaying remarkable homogeneity of their fluorescence intensity (Figure 5b,c). Importantly, the overall (donor + acceptor) intensity recorded per NP-probe was similar to that for control NP-T20 (without acceptor), being 100-fold higher compared to QDdot-605 excited at optimal (470 nm) wavelength (Figure 5b,c, Table S3). This unprecedented brightness for a FRET biosensor is explained by high overall fluorescence quantum yield of NP-probe (46\%) and 3200 rhodamine dyes inside nanoantenna with total molar extinction coefficient of $\sim 3.2 \times 10^{8} \mathrm{M}^{-1} \mathrm{~cm}^{-1}$ at $550 \mathrm{~nm}\left(\mathrm{vs} .1 .1 \times 10^{6} \mathrm{M}^{-1} \mathrm{~cm}^{-}\right.$ ${ }^{1}$ for QDot-605 at $488 \mathrm{~nm}$ ) that serve as giant ensemble of energy donors.

To evaluate FRET signal at the single particle level, the images of NPs were recorded simultaneously at the green (donor) and red (acceptor) channels. It can be seen that NP-probe showed similar intensities at these two channels and appeared mostly yellow in the overlay (Figure 5d), whereas control NP-T20 showed signal only in the donor channel (Figure 6a). These results provide clear evidence that, after immobilization at the surface, our NP-probe preserved strong FRET, as in the spectroscopy measurements (Figure 3c). Then, to evaluate antenna effect at the single-particle level, we compared emission of the acceptor excited via nanoantenna (at $550 \mathrm{~nm}$ ) vs that directly excited at $640 \mathrm{~nm}$. Remarkably, 50-fold higher excitation power density (irradiance) at $640 \mathrm{~nm}$ was required to achieve emission intensity comparable to that excited at $550 \mathrm{~nm}$ (Figure 5d). Quantitative image analysis revealed $75 \pm 30$ fold amplification of acceptor emission (Figure 5e), in line with the spectroscopic data in solution. We should note that, this is the first report where this high amplification is reported at the single particle level for a biosensor. Previous report that used QDot as a FRET donor for NA detection at the single particle level did not exploit light-harvesting concept because a large number of acceptors per particle $(\sim 50)$ was required to achieve efficient FRET. ${ }^{14 \mathrm{~b}}$ Indeed, the ratio of extinction coefficients of QDot-605 $\left(1.1 \times 10^{6} \mathrm{M}^{-1} \mathrm{~cm}^{-1}\right.$ at $\left.488 \mathrm{~nm}\right)$ to 50 acceptors $\left(2.5 \times 10^{5} \times 50=1.25 \times 10^{7} \mathrm{M}^{-1} \mathrm{~cm}^{-1}\right)$ is 0.09 , suggesting that excitation through QDot-605 is less efficient than direct excitation of the acceptors. In case of our nanoantenna, the ratio of extinction coefficients of nanoantenna and 23 acceptors at the used excitation wavelengths $(550$ and $640 \mathrm{~nm})$ is $3.2 \times 10^{8} / 4.5 \times 10^{6}=71$, in agreement with the amplification 
values measured at the single-particle level. The amplification at the single-particle level within a biosensor was reported very recently for a plasmonic system using DNA origami, where the average amplification was $7.3 .^{25}$
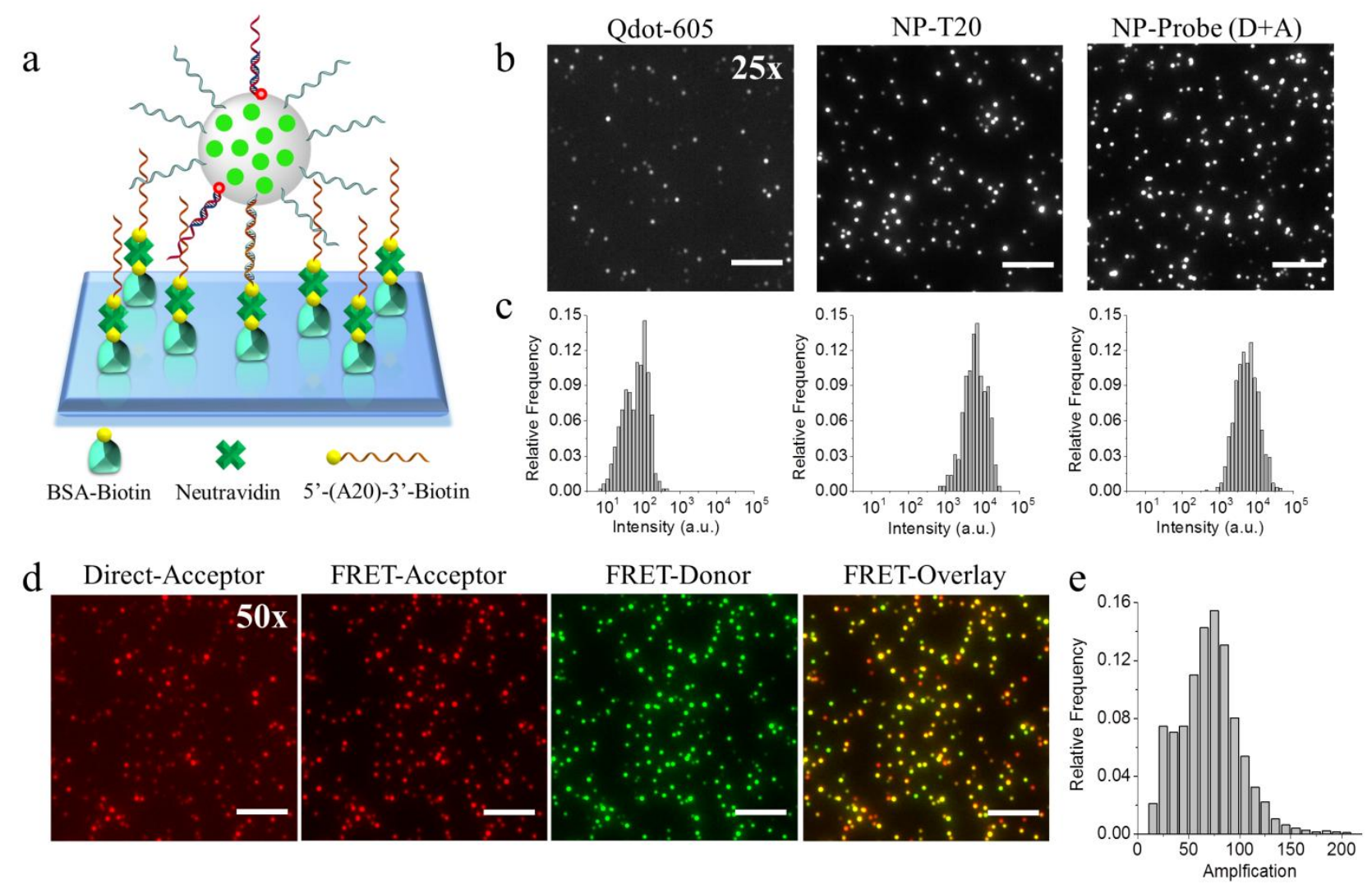

Figure 5. Single-particle imaging of immobilized nanoprobes. (a) Scheme of nanoprobes immobilization on glass surface modified with BSA-biotin, neutravidin, and A20-biotin. The interaction with the surface occurs due to hybridization of A20-biotin with T20 of NP. (b) Wide-field fluorescence microscopy of the immobilized NPs: quantum dots (QDot-605) excited at $470 \mathrm{~nm}$, control NP-T20 and nanoprobe (sum of donor and acceptor channels is shown). To obtain comparable signals excitation power density for QDot-605 was 25-fold higher than that for NP-T20 and nanoprobe: 14 vs $0.56 \mathrm{~W} \mathrm{~cm}^{-}$ ${ }^{2}$, respectively. (c) Histogram of single-particle intensity distribution for these three types of NPs. At least 1000 NPs were analyzed in each case. (d) Wide-field microscopy images of the nanoprobe at the acceptor (direct excitation at $640 \mathrm{~nm}$ ), antenna-amplified acceptor (FRET-Acceptor), donor (FRETdonor) and merged FRET-Acceptor and donor channels (at the same intensity scale). The excitation of nanoprobe (FRET-Donor and FRET-Acceptor) was at $550 \mathrm{~nm}$ with excitation power density $0.4 \mathrm{~W} \mathrm{~cm}$ ${ }^{2}$, while direct excitation of acceptor was done at $640 \mathrm{~nm}$ using 50 -fold higher power density $(20 \mathrm{~W} \mathrm{~cm}$ ${ }^{2}$ ). Signals from FRET-Donor and FRET-Acceptor were recorded at $<640$ and $>640 \mathrm{~nm}$, respectively. Integration time was systematically $200 \mathrm{~ms}$. (e) Amplification of FRET-Acceptor emission (antenna effect) by the nanoprobe at the single-particle level presented as a distribution histogram. At least 1500 NPs were analyzed. 
$\mathrm{a}$

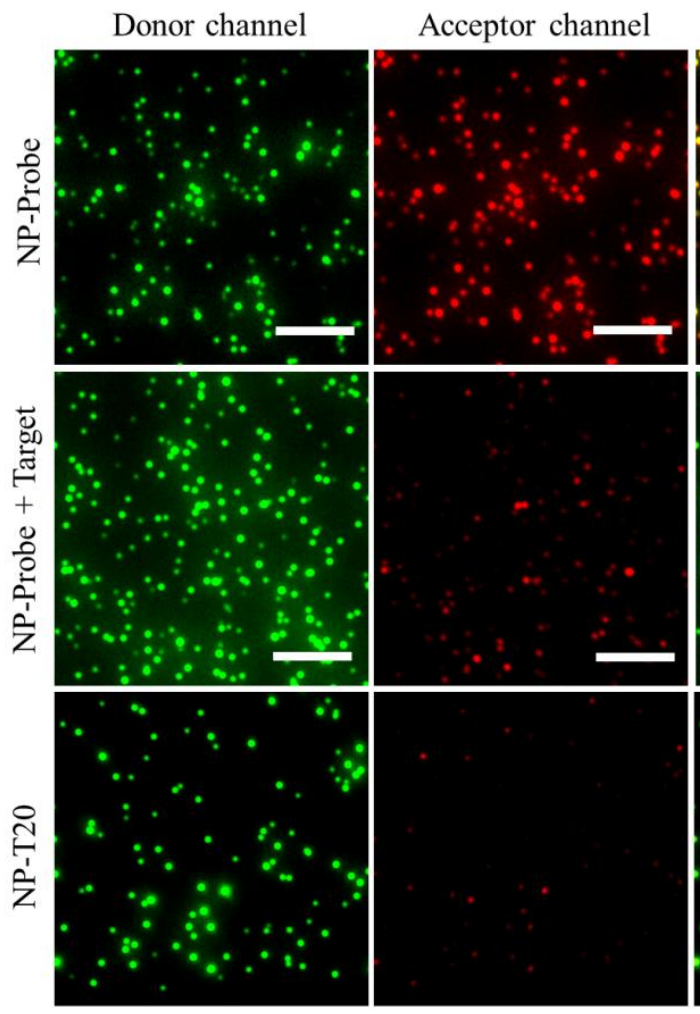

Overlay
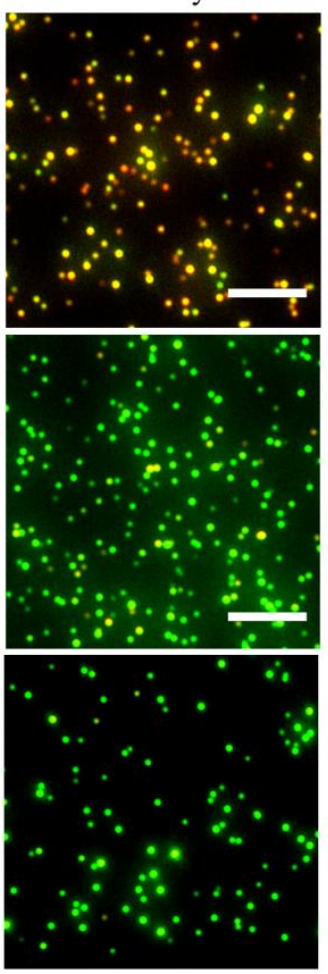

b Histogram Ratio $\frac{A}{A+D}$
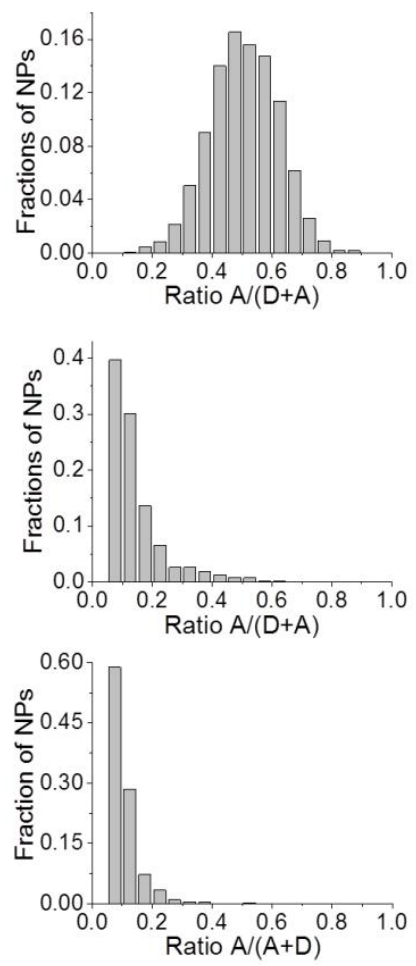

Figure 6. Response of surface-immobilized NP-probe to the NA target at the single-particle level. (a) Wide-field FRET images (donor channel, acceptor channels and overlay) and (b) corresponding histograms of relative FRET efficiency, $\mathrm{A} /(\mathrm{A}+\mathrm{D})$, of the NP-probe (first row), nanoprobe after incubation with $100 \mathrm{pM}$ survivin NA target for $20 \mathrm{~h}$ at $4^{\circ} \mathrm{C}$ (second row) and control NP-T20 (third row). All channels are represented at the same intensity scale. The excitation wavelength was at $550 \mathrm{~nm}$ with $0.4 \mathrm{~W} \mathrm{~cm}^{-2}$ power density. Signals from the donor and the acceptor were recorded at $<640$ and $>640 \mathrm{~nm}$, respectively. A and D are the integrated densities of the signal from individual particles recorded at the acceptor and the donor channels, respectively.

Finally, we tested the response of our nanoprobe to the target at the single-particle level (Figure 6). After incubation of immobilized NP-probe with the target the emission in the red channel strongly decreased, in line with our observations by fluorescence spectroscopy. The semi-quantitative FRET efficiency parameter, $\mathrm{A} /(\mathrm{A}+\mathrm{D})$, decreased from 0.48 down to 0.20 (Figure $6 \mathrm{~b}$ ), reaching values close to that for the control NP-T20 without FRET acceptor (0.12). Immobilized NP-probe was able to detect NA target in the concentration range of $1-1000 \mathrm{pM}$ (Figure 7a and S11) with a remarkably low LOD of $0.25 \mathrm{pM}$ (down to $12.5 \times 10^{-18}$ moles in $50 \mu \mathrm{L}$ ). At low target concentration, the response of NP-probe was significantly improved for longer incubation times (Figure 7a), showing that sensitivity of our system was only limited by the kinetics of hybridization with the target NA. The latter is known to be on the time scale of hours for pM NA concentrations. ${ }^{36}$ Robust detection of 10 pM NA target at the singleparticle level was achieved in the presence of BSA, bovine and human serum (Figure 7b,c and S12), showing potential of our surface-immobilized NP-probe for development of future diagnostics assays. 
Finally, we should stress that $\sim 23$ hybridization events at the surface of the single NP-probe (corresponding to the number of TCS-Cy5 per NP) resulted in the color switching of a particle exhibiting the brightness of $>3000$ rhodamine dyes or $\sim 100$ QDot-605. This outstanding performance of the developed NP-probe has two important consequences. First, the observed color switching implies that, at the single particle level, the NP-probe could readily detect just a few copies of the nucleic acid target. Indeed, according to spectroscopy data, hybridization of 3-5 nucleotides can provide detectable change in the FRET signal (Figure 3h). Second, due to the signal amplification produced by light harvesting with $>3000$ dyes, the target nucleic acids could be detected at very low excitation power density $\left(0.4 \mathrm{~W} \mathrm{~cm}^{2}\right)$ of LED in an epi-fluorescence mode, which is $>100$ fold-lower than required in the single-molecule detection measurements. The use of low power significantly decreases the background noise and makes possible detection of a few copies of nucleic acids using relatively weak and inexpensive light sources and a simple imaging setup.
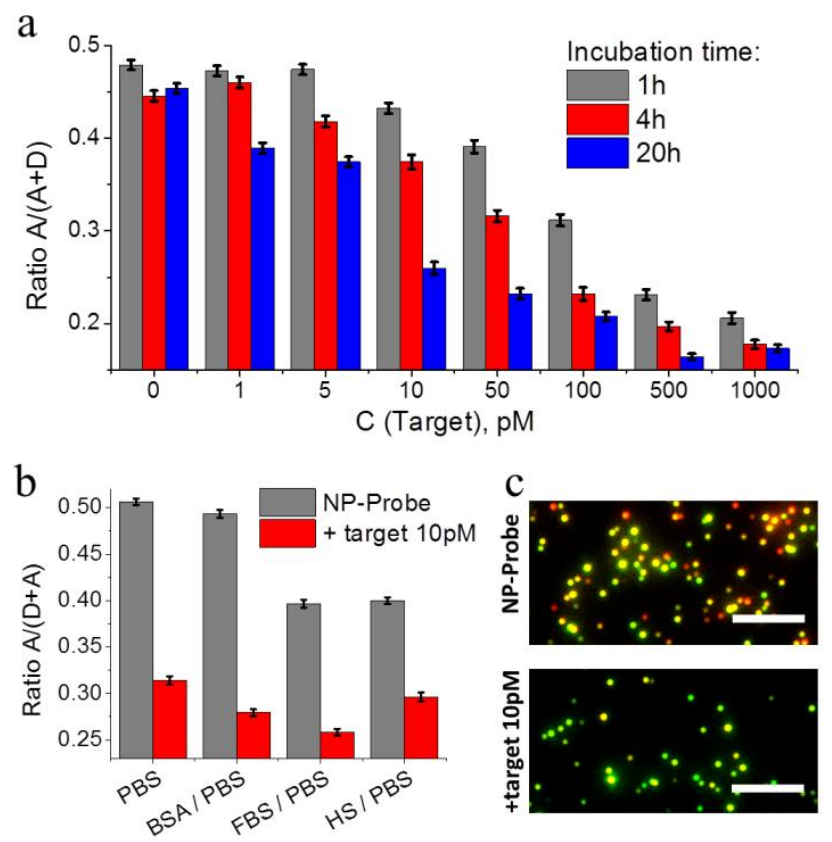

Figure 7. Single-particle evaluation of NP-probe ratiometric response in different conditions. (a) Ratiometric response of the immobilized NP-probe to different concentrations of survivin NA target after three different incubation times: 1 and $4 \mathrm{~h}$ at room temperature, and $20 \mathrm{~h}$ at $4{ }^{\circ} \mathrm{C}$. (b) Reponses of NP-probe $10 \mathrm{pM}$ NA target after $20 \mathrm{~h}$ incubation at $4{ }^{\circ} \mathrm{C}$ in different biological media: BSA, FBS and human serum (HS) in PBS. (a,b) The error bars are s.e.m. At least 1000 particles were analyzed for each condition. (c) Example of overlaid images of Donor and Acceptor channels for NP-probe incubated with $10 \mathrm{pM}$ NA target after $20 \mathrm{~h}$ incubation at $4{ }^{\circ} \mathrm{C}$. The excitation wavelength was $550 \mathrm{~nm}$ with power density of $0.4 \mathrm{~W} \mathrm{~cm}^{-2}$; integration time was $200 \mathrm{~ms}$. Scale bar, $5 \mu \mathrm{m}$.

\section{CONCLUSIONS}

Here, we introduce a concept of amplified fluorescence sensing based on giant light-harvesting ensemble of organic dyes in a polymeric nanoparticle (nanoantenna). This nanoantenna serves as super- 
efficient energy donor in a FRET-based nucleic acid detection assay, where a few hybridization events at the surface of the nanoparticle switch on/off energy transfer from thousands of dyes. As chemical modification of small polymeric nanoparticles with oligonucleotides remains a challenge, we developed an original strategy for functionalization of the polymeric nanoantennas. Bi-functional moiety bearing charged carboxylate and azide was grafted to a biocompatible hydrophobic polymer (PMMA-MA), which ensured (i) preparation of small nanoparticles $(\sim 40 \mathrm{~nm})$ by nanoprecipitation and (ii) effective exposure of the azide group for coupling with oligonucleotides by $\mathrm{Cu}$-free click reaction. Using this strategy, we functionalized the nanoantenna particles with simultaneously two oligonucleotides. The first one is the molecular recognition unit that after hybridization with the target nucleic acid (encoding a fragment of cancer marker survivin) removes the energy acceptor (Cy5 dye) from the nanoantenna surface, triggering the loss of FRET. The second one is a non-coding oligonucleotide, which is found to be essential for nanoparticle stability in biological media. The obtained nanoprobes display efficient FRET ( $>60 \%$ ) from $\sim 3200$ rhodamine-based donor dyes of nanoantenna to only 23 Cy5-acceptors. This efficient light-harvesting phenomenon results in the amplification of the acceptor emission (antenna effect) reaching 75 . The nanoprobe detects target nucleic acid by changing the ratio of its donor/acceptor intensity ratio and can distinguish down to 3-5 hybridizations at its surface. Our nanoantenna based probe is $\sim 100$-fold brighter than quantum dots (QDot-605) and $>2000$-fold brighter than analogous FRET-based molecular probe. To our knowledge, it is the first nanoparticle-based probe for biomolecules that combines this exceptional brightness and such high signal amplification. The nanoprobe operates in solution and on surfaces at the single-particle level delivering limit of detection of 5 and $0.25 \mathrm{pM}$, respectively, the latter being limited only by the hybridization kinetics. We expect that outstanding brightness and amplification characteristics of nanoantenna probes will greatly simplify detection of nucleic acids with low-cost portable fluorescence detection instruments, which is of highest importance for detection biomolecular markers of diseases in point of care diagnostics. Moreover, our functionalized organic nanoantenna, owing to the exceptional capacity to donate the energy from thousands of donors to few acceptors at its surface, can increase by orders of magnitude brightness of practically any FRET based detection assay.

\section{EXPERIMENTAL SECTION}

Oligonucleotides. Lyophilized single strand DNA sequences were purchased from IBA, dissolved in Milli-Q water, aliquoted and stored at $-20{ }^{\circ} \mathrm{C}$ for further experiments. The oligonucleotide sequences used in this study are shown below. SurC-DBCO, 5'-CCC AGC CTT CCA GCT CCT TGA -(DBCO)3'; T20-DBCO, 5'-TTT TTT TTT TTT TTT TTT TT-(DBCO)-3'; TCS-Cy5, 5'-(Cy5)-TCA AGG AGC TGG-3'; Target, 5'-CAA GGA GCT GGA AGG CTG GG-3'; single mismatch, 5'-CAA GCA GCT GGA AGG CTG GG-3'; two mismatches, 5'-CAA GCA GCT GGA AGC CTG GG-3'; three mismatches, 5'-CAA GCA GCT CGA AGC CTG GG-3'; A20-Biotin, 5' -(BIO)-AAA AAA AAA AAA AAA AAA AA-3'. 
Nanoparticle preparation. Protocol for diluted NPs: $50 \mu \mathrm{L}$ of the PMMA-AspN3 polymer solution in acetonitrile $\left(1 \mathrm{mg} \mathrm{mL}^{-1}\right.$ containing $\mathrm{R} 18 / \mathrm{F} 5-\mathrm{TPB}$ at $30 \mathrm{wt} \%$ relative to the polymer) were added quickly using a micropipette to $450 \mu \mathrm{L}$ of $20 \mathrm{mM}$ phosphate buffer, $\mathrm{pH} 7.4$ at $21{ }^{\circ} \mathrm{C}$ under shaking (Thermomixer comfort, Eppendorf, $1100 \mathrm{rpm}$ ). The particle solution was then quickly diluted 5-fold with the same buffer. For preparation of NPs functionalized with DNA the protocol was modified to increase NPs concentration: $100 \mu \mathrm{L}$ of the PMMA-AspN3 polymer solution in acetonitrile $\left(2 \mathrm{mg} \mathrm{mL}^{-1}\right.$ with $30 \mathrm{wt} \% \mathrm{R} 18 / \mathrm{F} 5-\mathrm{TPB}$ relative to the polymer) were then added quickly using a micropipette to 900 $\mu \mathrm{L}$ the same buffer under shaking with Thermomixer $(1,100 \mathrm{rpm})$. Then, the residues of acetonitrile were evaporated.

NP-probe synthesis. Aliquots of DNA ( $3 \mu \mathrm{M}$ SurC-DBCO, with or without $20 \mu \mathrm{M}$ T20-DBCO) were added to $300 \mu \mathrm{L}$ of nanoparticles (concentration of azide groups were $\sim 26 \mu \mathrm{M}$ ). The reaction was mixed and kept overnight at $40{ }^{\circ} \mathrm{C}$ in Thermomixer without shaking protected from light. Then the reaction was cooled down to room temperature. For annealing with TCS-Cy5, the aliquot of TCS-Cy5 in ratio 1:1 with SurC-DBCO was added and mixture was heated to $70{ }^{\circ} \mathrm{C}$ in water bath for $3 \mathrm{~min}$. To complete hybridization the reaction was cooled down to room temperature and kept in the dark for $2 \mathrm{~h}$. Then the mixture was diluted with $20 \mathrm{mM}$ phosphate buffer containing $12 \mathrm{mM} \mathrm{MgCl}_{2}$ and $30 \mathrm{mM} \mathrm{NaCl}$ to $4 \mathrm{~mL}$ and purified by centrifugation using centrifuge filters (Amicon, $0.5 \mathrm{ml}, 100 \mathrm{k}$ ) on $1000 \mathrm{~g}$ at $20{ }^{\circ} \mathrm{C}$ for 2 $\mathrm{min}$. The procedure of centrifugation was repeated 5 times to remove the non-reacted oligonucleotides. The obtained NP-probes in volume of $1 \mathrm{~mL}$ were kept in the dark at $4{ }^{\circ} \mathrm{C}$.

Detection of survivin nucleic acid target. For detection in solution the NP-Probe was diluted in 20 $\mathrm{mM}$ phosphate buffer containing $30 \mathrm{mM} \mathrm{NaCl}$ and $12 \mathrm{mM} \mathrm{MgCl}_{2}$ to a desired concentration (corresponding to TCS-Cy5) and aliquot of target oligonucleotide was added. Before measurements the mixture was incubated in the dark at $4{ }^{\circ} \mathrm{C}$ for $20 \mathrm{~h}$, unless specified. For detection of target NA on surfaces, the aliquot of the NA target was added to the LabTek chamber with immobilized NP-Probes. The mixture was kept in the dark at the room temperature for 1 and $4 \mathrm{~h}$ incubation or at $4{ }^{\circ} \mathrm{C}$ for $20 \mathrm{~h}$ incubation.

For NA detection in different biological media, the PBS and OptiMEM were adjusted to $12 \mathrm{mM} \mathrm{MgCl} 2$ concentrations and further used for sample preparations. BSA was diluted to $0.5 \mathrm{mg} / \mathrm{mL}$ concentration in PBS. The deoxyribonucleic acid sodium salt from calf thymus (CT-DNA) was dissolved to concentration of $2 \mathrm{mg} / \mathrm{mL}$ in water and kept overnight at $4{ }^{\circ} \mathrm{C}$ for complete dissolution. Then, it was heated for $3 \mathrm{~min}$ at $90{ }^{\circ} \mathrm{C}$ followed by rapid transfer into ice bath. For measurements it was diluted to concentration of $10 \mu \mathrm{g} / \mathrm{mL}$ in PBS. Foetal bovine serum (FBS) and Human Serum were heat-inactivated by exposing them for $30 \mathrm{~min}$ to $56{ }^{\circ} \mathrm{C}$ before they were used. After that the FBS and Human Serum were diluted to $10 \%$ concentration in OptiMEM. For measurements in solution the NP-Probe was diluted in the corresponding media and the target oligonucleotide was added. Before the measurements were made, the mixtures of NP-Probe with and without target oligonucleotide were incubated in the dark at $4{ }^{\circ} \mathrm{C}$ for a given time. For the measurements on the surface, $300 \mu \mathrm{L}$ of the media was mixed with aliquot of the target oligonucleotide and then the mixture was added to the LabTek chamber with immobilized NP-Probes. Prepared mixtures were incubated for 20 hours at $4{ }^{\circ} \mathrm{C}$ before measurements. 


\section{ASSOCIATED CONTENT}

\section{Supporting Information}

Materials and methods section, supporting tables and figures.

This material is available free of charge via the Internet at http://pubs.acs.org.

\section{AUTHOR INFORMATION}

\section{Corresponding Author}

* andrey.klymchenko@unistra.fr

\section{Notes}

A European patent application $\mathrm{n}^{\circ} 18305253.9$ has been filed.

\section{ACKNOWLEDGMENT}

This work was supported by the European Research Council ERC Consolidator grant BrightSens 648528. We thank to C. Ruhlmann from FRISBI platform for help with electron microscopy, A. Reisch for fruitful discussions and J. Valanciunaite for reading the manuscript.

\section{REFERENCES}

(1) Grimm, J. B.; English, B. P.; Chen, J. J.; Slaughter, J. P.; Zhang, Z. J.; Revyakin, A.; Patel, R.; Macklin, J. J.; Normanno, D.; Singer, R. H.; Lionnet, T.; Lavis, L. D. Nat. Methods 2015, 12, 244.

(2) Scrimin, P.; Prins, L. J. Chem. Soc. Rev. 2011, 40, 4488.

(3) (a) Choi, H. M. T.; Chang, J. Y.; Trinh, L. A.; Padilla, J. E.; Fraser, S. E.; Pierce, N. A. Nat. Biotechnol. 2010, 28, 1208. (b) Ali, M. M.; Li, F.; Zhang, Z.; Zhang, K.; Kang, D.-K.; Ankrum, J. A.; Le, X. C.; Zhao, W. Chem. Soc. Rev. 2014, 43, 3324.

(4) (a) Thomas, S. W.; Joly, G. D.; Swager, T. M. Chem. Rev. 2007, 107, 1339. (b) Wu, W. B.; Bazan, G. C.; Liu, B. Chem 2017, 2, 760. (c) Rochat, S.; Swager, T. M. ACS Appl. Mater. Interfaces 2013, 5, 4488. (d) Jiang, Y.; McNeill, J. Chem. Rev. 2017, 117, 838. (e) Chen, L. H.; McBranch, D. W.; Wang, H. L.; Helgeson, R.; Wudl, F.; Whitten, D. G. Proc. Natl. Acad. Sci. U.S.A. 1999, 96, 12287.

(5) (a) Novotny, L.; van Hulst, N. Nat. Photonics 2011, 5, 83. (b) Acuna, G. P.; Moller, F. M.; Holzmeister, P.; Beater, S.; Lalkens, B.; Tinnefeld, P. Science 2012, 338, 506. (c) Anker, J. N.; Hall, W. P.; Lyandres, O.; Shah, N. C.; Zhao, J.; Van Duyne, R. P. Nat. Mater. 2008, 7, 442.

(6) (a) Howes, P. D.; Chandrawati, R.; Stevens, M. M. Science 2014, 346, 1247390. (b) Wolfbeis, O. S. Chem. Soc. Rev. 2015, 44, 4743. (c) Chinen, A. B.; Guan, C. M.; Ferrer, J. R.; Barnaby, S. N.; Merkel, T. J.; Mirkin, C. A. Chem. Rev. 2015, 115, 10530.

(7) (a) Alivisatos, P. Nat. Biotechnol. 2004, 22, 47. (b) Wegner, K. D.; Hildebrandt, N. Chem. Soc. Rev. 2015, 44, 4792. 
(8) (a) Bonacchi, S.; Genovese, D.; Juris, R.; Montalti, M.; Prodi, L.; Rampazzo, E.; Zaccheroni, N. Angew. Chem. Int. Ed. 2011, 50, 4056. (b) Lee, J. E.; Lee, N.; Kim, T.; Kim, J.; Hyeon, T. Acc. Chem. Res. 2011, 44, 893.

(9) (a) Mei, J.; Leung, N. L. C.; Kwok, R. T. K.; Lam, J. W. Y.; Tang, B. Z. Chem. Rev. 2015, 115, 11718. (b) Li, K.; Liu, B. Chem. Soc. Rev. 2014, 43, 6570.

(10) (a) Wu, C.; Chiu, D. T. Angew. Chem. Int. Ed. 2013, 52, 3086. (b) Pecher, J.; Mecking, S. Chem. Rev. 2010, 110, 6260.

(11) Reisch, A.; Klymchenko, A. S. Small 2016, 12, 1968.

(12) (a) Medintz, I. L.; Clapp, A. R.; Mattoussi, H.; Goldman, E. R.; Fisher, B.; Mauro, J. M. Nat. Mater. 2003, 2, 630. (b) Hildebrandt, N.; Spillmann, C. M.; Algar, W. R.; Pons, T.; Stewart, M. H.; Oh, E.; Susumu, K.; Diaz, S. A.; Delehanty, J. B.; Medintz, I. L. Chem. Rev. 2017, 117, 536. (c) Sapsford, K. E.; Berti, L.; Medintz, I. L. Angew. Chem. Int. Ed. 2006, 45, 4562. (d) Clapp, A. R.; Medintz, I. L.; Uyeda, H. T.; Fisher, B. R.; Goldman, E. R.; Bawendi, M. G.; Mattoussi, H. J. Am. Chem. Soc. 2005, 127, 18212. (e) Boeneman, K.; Mei, B. C.; Dennis, A. M.; Bao, G.; Deschamps, J. R.; Mattoussi, H.; Medintz, I. L. J. Am. Chem. Soc. 2009, 131, 3828.

(13) Genovese, D.; Rampazzo, E.; Bonacchi, S.; Montalti, M.; Zaccheroni, N.; Prodi, L. Nanoscale 2014, 6, 3022.

(14) (a) Clapp, A. R.; Medintz, I. L.; Mauro, J. M.; Fisher, B. R.; Bawendi, M. G.; Mattoussi, H. J. Am. Chem. Soc. 2004, 126, 301. (b) Zhang, C. Y.; Yeh, H. C.; Kuroki, M. T.; Wang, T. H. Nat. Mater. 2005, $4,826$.

(15) Reisch, A.; Trofymchuk, K.; Runser, A.; Fleith, G.; Rawiso, M.; Klymchenko, A. S. ACS Appl. Mater. Interfaces 2017, 9, 43030.

(16) (a) Reisch, A.; Didier, P.; Richert, L.; Oncul, S.; Arntz, Y.; Mely, Y.; Klymchenko, A. S. Nat. Commun. 2014, 5, 4089. (b) Andreiuk, B.; Reisch, A.; Lindecker, M.; Follain, G.; Peyrieras, N.; Goetz, J. G.; Klymchenko, A. S. Small 2017, 13, 1701582.

(17) Scholes, G. D.; Fleming, G. R.; Olaya-Castro, A.; van Grondelle, R. Nat. Chem. 2011, 3, 763.

(18) Trofymchuk, K.; Reisch, A.; Didier, P.; Fras, F.; Gilliot, P.; Mely, Y.; Klymchenko, A. S. Nat. Photonics 2017, 11, 657.

(19) Puchkova, A.; Vietz, C.; Pibiri, E.; Wunsch, B.; Paz, M. S.; Acuna, G. P.; Tinnefeld, P. Nano Lett. 2015, 15,8354 .

(20) (a) Brown, P. O.; Botstein, D. Nat. Genet. 1999, 21, 33. (b) Tyagi, S. Nat. Methods 2009, 6, 331. (c) Zheng, J.; Yang, R. H.; Shi, M. L.; Wu, C. C.; Fang, X. H.; Li, Y. H.; Li, J. H.; Tan, W. H. Chem. Soc. Rev. 2015, 44, 3036. (d) Jung, C.; Ellington, A. D. Acc. Chem. Res. 2014, 47, 1825. (e) Seferos, D. S.; Giljohann, D. A.; Hill, H. D.; Prigodich, A. E.; Mirkin, C. A. J. Am. Chem. Soc. 2007, 129, 15477. (f) Cutler, J. I.; Auyeung, E.; Mirkin, C. A. J. Am. Chem. Soc. 2012, 134, 1376. (g) Labib, M.; Mohamadi, R. M.; Poudineh, M.; Ahmed, S. U.; Ivanov, I.; Huang, C.-L.; Moosavi, M.; Sargent, E. H.; Kelley, S. O. Nat. Chem. 2018, 10, 489. (h) He, L.; Lu, D. Q.; Liang, H.; Xie, S. T.; Luo, C.; Hu, M. M.; Xu, L. J.; Zhang, X. B.; Tan, W. H. ACS Nano 2017, 11, 4060.

(21) Nolan, T.; Hands, R. E.; Bustin, S. A. Nat. Protoc. 2006, 1, 1559.

(22) (a) Dirks, R. M.; Pierce, N. A. Proc. Natl. Acad. Sci. U.S.A. 2004, 101, 15275. (b) Huang, J.; Wang, H.; Yang, X. H.; Quan, K.; Yang, Y. J.; Ying, L.; Xie, N. L.; Ou, M.; Wang, K. M. Chem. Sci. 2016, 7, 3829. (c) Wu, C. C.; Cansiz, S.; Zhang, L. Q.; Teng, I. T.; Qiu, L. P.; Li, J.; Liu, Y.; Zhou, C. S.; Hu, R.; Zhang, T.; Cui, C.; Cui, L.; Tan, W. H. J. Am. Chem. Soc. 2015, 137, 4900.

(23) (a) Gaylord, B. S.; Heeger, A. J.; Bazan, G. C. Proc. Natl. Acad. Sci. U.S.A. 2002, 99, 10954. (b) Wang, Y. S.; Liu, B. Chem. Commun. 2007, 3553. (c) Dore, K.; Dubus, S.; Ho, H. A.; Levesque, I.; Brunette, M.; Corbeil, G.; Boissinot, M.; Boivin, G.; Bergeron, M. G.; Boudreau, D.; Leclerc, M. J. Am. Chem. Soc. 2004, 126, 4240.

(24) Wang, S.; Gaylord, B. S.; Bazan, G. C. J. Am. Chem. Soc. 2004, 126, 5446. 
(25) Ochmann, S. E.; Vietz, C.; Trofymchuk, K.; Acuna, G. P.; Lalkens, B.; Tinnefeld, P. Anal. Chem. 2017, 89, 13000.

(26) (a) Alemdaroglu, F. E.; Herrmann, A. Org. Biomol. Chem. 2007, 5, 1311. (b) Wilks, T. R.; O'Reilly, R. K. Sci. Rep. 2016, 6, 39192.

(27) (a) Watson, K. J.; Park, S. J.; Im, J. H.; Nguyen, S. T.; Mirkin, C. A. J. Am. Chem. Soc. 2001, 123, 5592. (b) Jia, F.; Lu, X. G.; Tan, X. Y.; Zhang, K. Chem. Commun. 2015, 51, 7843. (c) Rush, A. M.; Nelles, D. A.; Blum, A. P.; Barnhill, S. A.; Tatro, E. T.; Yeo, G. W.; Gianneschi, N. C. J. Am. Chem. Soc. 2014, 136, 7615 .

(28) (a) Banga, R. J.; Krovi, S. A.; Narayan, S. P.; Sprangers, A. J.; Liu, G. L.; Mirkin, C. A.; Nguyen, S. T. Biomacromolecules 2017, 18, 483. (b) Farokhzad, O. C.; Jon, S. Y.; Khademhosseini, A.; Tran, T. N. T.; LaVan, D. A.; Langer, R. Cancer Res. 2004, 64, 7668. (c) Zhu, S. S.; Xing, H.; Gordiichuk, P.; Park, J.; Mirkin, C. A. Adv. Mater. 2018, 30, 1707113.

(29) Reisch, A.; Runser, A.; Arntz, Y.; Mely, Y.; Klymchenko, A. S. ACS Nano 2015, 9, 5104.

(30) Olie, R. A.; Simoes-Wust, A. P.; Baumann, B.; Leech, S. H.; Fabbro, D.; Stahel, R. A.; Zangemeister-Wittke, U. Cancer Res. 2000, 60, 2805.

(31) (a) Prigodich, A. E.; Randeria, P. S.; Briley, W. E.; Kim, N. J.; Daniel, W. L.; Giljohann, D. A.; Mirkin, C. A. Anal. Chem. 2012, 84, 2062. (b) Prigodich, A. E.; Seferos, D. S.; Massich, M. D.; Giljohann, D. A.; Lane, B. C.; Mirkin, C. A. ACS Nano 2009, 3, 2147.

(32) Jewett, J. C.; Bertozzi, C. R. Chem. Soc. Rev. 2010, 39, 1272.

(33) Lauer, M. H.; Vranken, C.; Deen, J.; Frederickx, W.; Vanderlinden, W.; Wand, N.; Leen, V.; Gehlen, M. H.; Hofkens, J.; Neely, R. K. Chem. Sci. 2017, 8, 3804.

(34) Woller, J. G.; Hannestad, J. K.; Albinsson, B. J. Am. Chem. Soc. 2013, 135, 2759.

(35) (a) Søren, P.; Marcus, W. L. ChemBioChem 2012, 13, 1990. (b) Nir, E.; Michalet, X.; Hamadani, K. M.; Laurence, T. A.; Neuhauser, D.; Kovchegov, Y.; Weiss, S. J. Phys. Chem. B 2006, 110, 22103.

(36) Zhang, J. X.; Fang, J. Z.; Duan, W.; Wu, L. R.; Zhang, A. W.; Dalchau, N.; Yordanov, B.; Petersen, R.; Phillips, A.; Zhang, D. Y. Nat. Chem. 2017, 10, 91. 


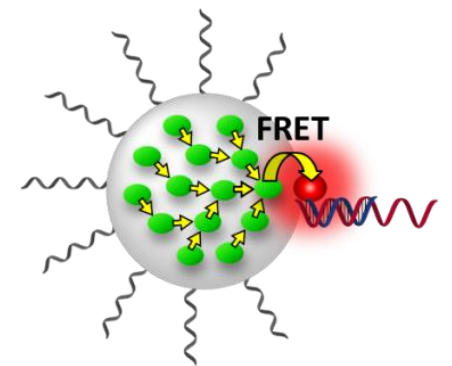

Light-harvesting in 40-nm antenna: 3200 donors $\Rightarrow 23$ acceptors

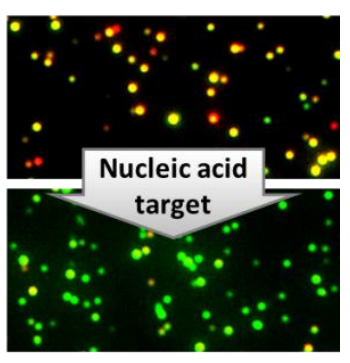

100-fold brighter than Qdot-605 